\title{
Orchestrating Directional Molecular Motions: Kinetically Controlled Supramolecular Pathways of a Helical Host on Rod-like Guests
}

\author{
Xiang Wang, Barbara Wicher, ${ }^{\dagger}$ Yann Ferrand, ${ }^{*}$ and Ivan Huc* \\ CBMN laboratory, Univ. Bordeaux, CNRS, IPB, Institut Européen de Chimie Biologie, 2 rue Escarpit 33607 Pessac, France.
}

\begin{abstract}
KEYWORDS: foldaxane, pseudo-rotaxane, foldamer, double helix, dynamic self-assembly, molecular recognition, directional
\end{abstract} motion, molecular shuttle, supramolecular pathways.

\begin{abstract}
An aromatic oligoamide sequence was designed to fold and self-assemble into a double helical host having a cylindrical cavity complementary to linear oligo-carbamates guests. Formation of helical pseudo-rotaxane complexes - foldaxanes - between the host and guests having binding stations of different affinities was evidenced by NMR and x-ray crystallography. Rod-like guests possessing two or three binding stations, long alkyl or oligoethylene glycol spacers or bulky barriers in-between the binding stations, and a single bulky stopper at one end, were synthesized. Kinetic investigations of the threading and translation of the double helix along multistation rods were monitored by ${ }^{1} \mathrm{H}$ NMR. Results show that multiple events may occur upon sliding of the host from the non-bulky end of the rod to reach the thermodynamically most stable state before unfolding-mediated dissociation has time to take place, including binding on intermediate stations and rapid sliding along non-binding spacers. Conversely, installing a kinetic barrier that blocks sliding allows for the deliberate integration of a helix dissociation re-association step in the supramolecular trajectory. Typical sliding processes can be monitored over the course of hours whereas steps involving unwinding/rewinding of the helix proceeded over the course of days. These results further demonstrate the interest of foldaxanes to design complex sequences of supramolecular events within networks of equilibria through the adjustment of the kinetics of the individual steps involved.
\end{abstract}

\section{INTRODUCTION}

A majority of the self-assembled artificial supramolecular architectures described to date form under thermodynamic control. Yet, a growing number of examples have been reported where kinetic control is essential to produce particular structures. ${ }^{1}$ Controlling the kinetics of self-assembly processes is important to create artificial molecular machines ${ }^{2}$ capable of directional motions. It is indeed the rates at which concommittent events take place that eventually determine in which order these events occur. Orchestrating the time scales of various molecular associations, dissociations and motions may thus allow for the design of sequences of events in a certain order between an initial state and a thermodynamically more stable state through a defined supramolecular trajectory. In the following, we report the design and characterization of such kinetically controlled supramolecular trajectories in the context of the association of aromatic amide helical foldamers with oligo-alkycarbamate rod-like guests to form helix-rod hostguest complexes termed foldaxanes. Foldaxane subcomponents may undergo several simultaneous dynamic processes. The generation of well-defined supramolecular pathways therefore required the integration of multiple parameters including foldamer helix folding; foldamer self-association into double helical dimers; equilibrium between parallel and anti-parallel forms of the duplexes; stabilization of the anti-parallel duplexes upon binding to rod-like guests to form foldaxane structures; availability of two foldaxane formation mechanisms (threading or direct winding around the rod) to create kinetic gradients; and availability of binding stations on the rods having different affinities for helix hosts to create thermodynamic gradients.

Foldaxane translation along molecular rods reproduce in model systems an important aspect of biomolecular machinery: the segregation on different timescales of several potentially reversible events. For instance biomolecular machines' subcomponents - proteins or nucleic acids - adopt folded conformation that are long lived, i.e. that do not undergo frequent spontaneously unfolding. Furthermore, these components self-assemble into larger supra-structures, e.g. the ribosome and multimeric protein complexes such as ATP synthase, that also are long lived and do not undergo spontaneous disassembly in the time frame required for their work regime. Thus, multiple motions can be implemented such as the disassembly of nucleic acid double helices by helicase, the transport of cargos within the cell by kinesin, or even the motility of entire cells through the motion of a flagellum as in spermatozoids on timescales much shorter than the assembly and dissociation time scales of the sub-components.

Translational molecular motions - typically the directional progression of a molecular shuttle along a molecular track - have been the object of particular attention. For example, the elucidation of the kinesin I motion mechanism ${ }^{3}$ inspired the development of artificial molecular walkers, ${ }^{4,5}$ designed to undergo a directional motion on a single molecular track through repetitive operations powered by an external source of fuel. Translation is also involved in the catalytic activity of DNA polymerase, a protein assembly that forms a pseudorotaxane-like complex with a DNA strand and moves along it while synthetizing a complementary sequence. Somewhat related to DNA polymerase are some artificial architectures in which a directional motion follows the threading of a macrocyclic molecule at one extremity of a rod possessing a stopper at the other extremity. Thus, pseudorotaxanes based on cyclodextrins ${ }^{6}$ or other macrocyclic ${ }^{7}$ architectures have been extensively used for the threading on oligomers or polymers. Some of these designs have even been shown to perform processive catalysis along the polymer track. ${ }^{8}$

Our group has shown that multi-turn helical aromatic oligoamide foldamers may wind around dumbbell shape molecules and form stable pseudo-rotaxane complexes termed foldaxanes $^{9}$ akin to other helix-rod host-guest complexes. ${ }^{10}$ 
These helical molecular tapes slowly wrap around oligocarbamate dumbbell rods then slide rapidly along them between two adjacent binding stations. The segregation of winding and sliding motion kinetics allowed for the construction of self-assembled molecular pistons ${ }^{9 a, b}$ without having to rest on permanently interlocked architectures. ${ }^{11}$ Herein, we report on our efforts at extending the scope of shuttling motions of helical aromatic oligoamide foldaxanes. We find that several sliding steps and processes - e.g. transient stops on intermediate binding stations and fast transit over rod segments deprived of binding stations - have time to proceed before dissociation through unwinding becomes significant. We also show that dissociation-reassociation can be purposely integrated in complex supramolecular trajectories to overcome a high kinetic barrier installed before a thermodynamic well that cannot be reached through sliding. These results further demonstrate the interest of foldaxanes to design complex sequences of supramolecular events within networks of equilibria through the adjustment of the kinetics of the individual steps involved.

A.
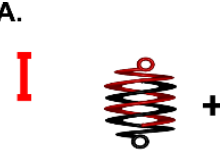

anti-parallel double helix

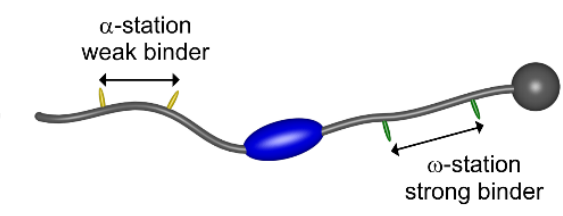

II

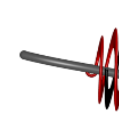

\section{III}

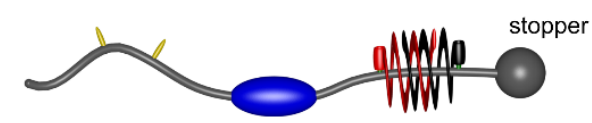

B.

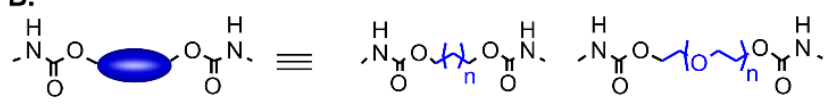

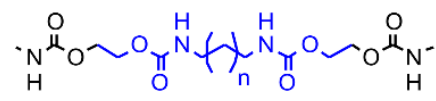

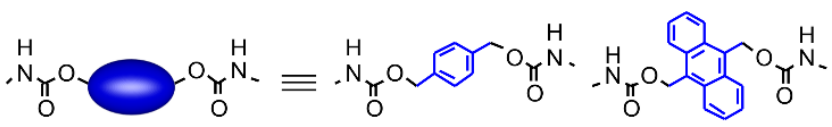

C. $\Longrightarrow$ : non bulky spacer

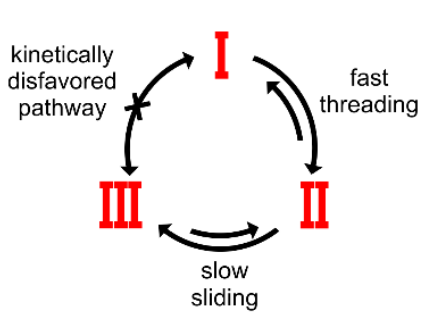

D.

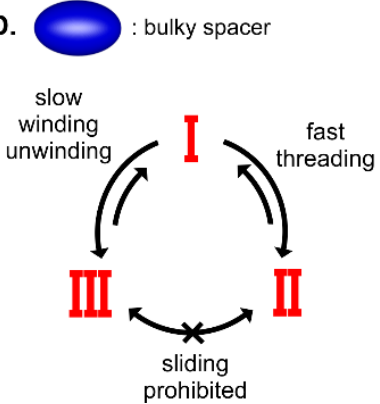

Figure 1. (A) Cartoon representation of the host-guest components and assembly. The host exists as an anti-parallel double helix. Rodlike guests possess carbonyl hydrogen bond acceptors (yellow or green sticks) defining two distinct binding stations having either a weak ( $\alpha$-station) or a strong affinity ( $\omega$-station) for the host. Each rod has a non-bulky terminus on which the host can be threaded, a bulky stopper at the other extremity, and a spacer (blue spheroid) of varying size connecting the two stations. I, II and III stand for three distinct supramolecular states of the host. (B) Formula of the different spacers used in this study. (C) Cycle showing the sliding pathway followed by the host with a non-bulky spacer. (D) Cycle showing the winding pathway followed by the host with a bulky spacer.

\section{RESULTS AND DISCUSSION}

Design and synthesis. We have previously shown that single and double helical aromatic oligoamide foldamers may bind to rod-like guests bearing carbamate groups separated by n-alkyl segments. ${ }^{\text {a-c }}$ Specifically, sequence $\mathbf{1}^{\text {cc }}$ (Figure 2A) folds and self-assembles as an anti-parallel double helix presenting two pyridine trimers, one at each extremity, that both act as hydrogen bond donors to complex the carbonyl functions of a guest (Figure 1A). The combined sixteen 8-fluoroquinoline monomers of the two strands define a one nanometer-long cylindrical cavity that accommodates n-alkanes. A match between the host and the guest lengths is required for optimal binding. Since $(\mathbf{1})_{2}$ is a dynamic structure capable of screwing-unscrewing motions, it may, to a limited extent, adjust its own length to that of the guest. ${ }^{9 b, c}$ Yet, upon varying guest length sufficiently, foldaxane thermodynamic stability varies to a large extent: binding constants ranging from $97 \mathrm{~L} \mathrm{~mol}^{-1}$ to $2.910^{4} \mathrm{~L} \mathrm{~mol}^{-1}$ have been measured upon increasing guest length from 6 to 12 methylene units. ${ }^{9 \mathrm{c}}$

We reasoned that several binding stations having an increasing affinity for $(\mathbf{1})_{2}$ may be placed sequentially along a multi-station rod so as to create a thermodynamic binding gradient. To induce the directional movement of a helix along that gradient, we planned to introduce several design features: (i) a bulky stopper may be placed at one extremity of the guest while the other extremity would consist of a short linear alkane that can quickly thread itself into the helix cavity. In this way, loading the helix on the rod by threading can be programmed to occur exclusively at a defined extremity regardless of the location of the best binding station; (ii) the affinity of $(\mathbf{1})_{2}$ for the first binding station ( $\alpha$-station) on the rod should be high enough to favor a mostly associated state under measurement conditions (typically NMR tube concentration) yet low enough not to hamper its sliding to a subsequent binding station along the rod; (iii) binding affinities should incrementally increase along the rod to bias the direction of the helix motion and to facilitate monitoring the helix progression through the successive appearance of sufficiently populated bound states; (iv) spacers of different nature, length and bulkiness may be placed in-between binding stations to control the sliding rates of the helix. Bulky spacers may eventually block the helix and impose other supramolecular pathways towards the most stable foldaxane; (v) when the helix reaches the last binding station ( $\omega$-station), the bulky stopper then prevents unslippage and makes the thermodynamically most stable state be kinetically stable as well.

A series of rod-like guests having up to three binding stations separated by spacers of varied length, polarity and bulkiness were designed and synthesized (Figure 2B,C,D). Synthetic routes and experimental details are provided in the supporting information. Typically, carbamate functions were produced by reaction of a primary alcohol or amine with 4-nitrophenyl chloroformate. The resulting activated 4-nitrophenyl carbamates reacted with primary alcohols to produce carbamates in boiling chloroform. Conversely, the resulting activated 4-nitrophenyl carbonates reacted with primary amines to produce carbamates 
at room temperature. Binding stations having, 7, 8 or 11 methylenes were used depending on the desired binding affinity. These affinities have been determined previously using single station rods ${ }^{9 c}$ (see below). Linear non hindered spacers between two binding stations were introduced to investigate whether they create a kinetic barrier to helix sliding along the rod. For instance, linear alkyl spacers of various length up to 18 methylenes $(\mathbf{2}, \mathbf{3}, \mathbf{4}$ and $\mathbf{5})$ and oligoethylene glycol spacers of various length up to 17 atoms $(\mathbf{2}, \mathbf{6}$ and 7$)$ were all placed between the same two stations. Note that the shortest spacer of compound $\mathbf{2}$ is the same for both series. In addition, two rods having three binding stations were prepared $(\mathbf{8}, \mathbf{9})$, which differ by the nature of the middle station, to generate more complex sliding pathways. In parallel rods bearing bulky spacers that hinder or forbid sliding motions were also synthesized. Benzene (10) and anthracene (11) were selected as bulky obstacles of varying size able to block the helix and to impose another supramolecular pathway, namely unwinding-winding, for the helix to reach the best binding station.

Self-assembly of 1 into double helical (1)2. The anti-parallel nature of $(\mathbf{1})_{2}$ was assessed both in solution and in the solid state. As shown by ${ }^{1} \mathrm{H}$ NMR, duplex $(\mathbf{1})_{2}$ exists as a mixture of parallel and antiparallel assemblies after purification on silica gel chromatography (Figure S1). Fortunately, the precipitation of the double helix from methanol provides $(\mathbf{1})_{2}$ exclusively in its antiparallel configuration. X-ray quality crystals of $(\mathbf{1})_{2}$ were obtained from the slow diffusion of $n$-hexane in a chloroform solution. The solid-state structure was solved and confirmed the antiparallel arrangement of $(\mathbf{1})_{2}$ as well as the existence of a one nanometer-long cylindrical cavity lined with fluorine atoms (Figure 3A-C). ${ }^{1} \mathrm{H}$ NMR monitoring of freshly dissolved antiparallel $(\mathbf{1})_{2}\left(4 \mathrm{mM}\right.$ in $\mathrm{CDCl}_{3}$ at $\left.17^{\circ} \mathrm{C}\right)$ showed that the parallel/antiparallel equilibrium was slowly reached after 10 hours (Figures S1 and S2, final ratio antiparallel/parallel $=63 / 37$ ). Only the antiparallel form of $(\mathbf{1})_{2}$ binds to rod-like guests. Thus, in the presence of the guests, the antiparallel form is further stabilized and the parallel form does not appear anymore, being disfavored both kinetically and thermodynamically. The main advantage of isolating the pure antiparallel duplex by the precipitation method mentioned above is thus to avoid a slow antiparallel to parallel duplex conversion to interfere with, and become the rate limiting factor of, the various transformations to be studied here.

Structural and thermodynamic study of foldaxane formation. Single station rods 12 and 13 possess one bulky stopper and one terminal $n$-butyl carbamate. Their binding to $(\mathbf{1})_{2}$ was assessed to be compared to the recently measured binding of dumbbell-shaped guests having two benzyl-carbamates. ${ }^{9 \mathrm{c}}$ The two rods possess 8 and 11 methylenes, respectively, between their carbamate functions. Upon adding guests $\mathbf{1 2}$ and $\mathbf{1 3}$ to a $\mathrm{CDCl}_{3}$ solution containing antiparallel $(\mathbf{1})_{2}$, single sets of wellresolved NMR signals were immediately observed as a result of the formation of $(\mathbf{1})_{2} \supset \mathbf{1 2}$ and (1) $)_{2} \supset \mathbf{1 3}$ (Figures S3 and S4). Despite the absence of stoppers on the rod, exchange between free $(\mathbf{1})_{2}$ and bound $(\mathbf{1})_{2}$ was found to be slow on the NMR time scale. Association constants for (1) $\supset$ 12 and (1) $)_{2} \supset \mathbf{1 3}$ were measured accurately through the integral ratios of the free and bound host amide resonances to be $K_{a}=6.410^{3}$ and $4.810^{4} \mathrm{~L}$ $\mathrm{mol}^{-1}$, respectively, at $17^{\circ} \mathrm{C}$ in $\mathrm{CDCl}_{3}$. These values are both about $40 \%$ higher than measured with bis-benzyl carbamate guests at $25^{\circ} \mathrm{C}$. ${ }^{9 \mathrm{c}}$ The ratio between these association constants was confirmed in a competition experiment (Figure S5): the addition of rod 13 ( 1 equiv.) to foldaxane $(\mathbf{1})_{2} \supset \mathbf{1 2}$ caused the dissociation of $(\mathbf{1})_{2}$ and its quick threading on $\mathbf{1 3}$ to form $(\mathbf{1})_{2} \supset \mathbf{1 3}$ (final ratio $\left[(\mathbf{1})_{2} \supset \mathbf{1 3}\right] /\left[(\mathbf{1})_{2} \supset \mathbf{1 2}\right]=79 / 21$ ). The 7.5 fold difference between the two affinities was considered to be sufficient to create a gradient of affinity on multistation rod molecules. As shown in the subsequent steps of this study, the design of single station rods $\mathbf{1 2}$ and $\mathbf{1 3}$ as models of individual stations on multistation rods 2-11 proved to be valid except in the case of rod 2. The two stations of $\mathbf{2}$ are close to each other and both possess an enhanced affinity for $\mathbf{1}$ (vide infra). In this case, $\mathbf{1 4}$ is a better model than $\mathbf{1 2}$ of the first binding station of $\mathbf{2}$.

\section{A. Aromatic oligoamide shuttle}

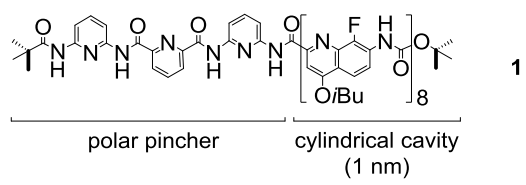

B. Oligocarbamate rods with non bulky spacers
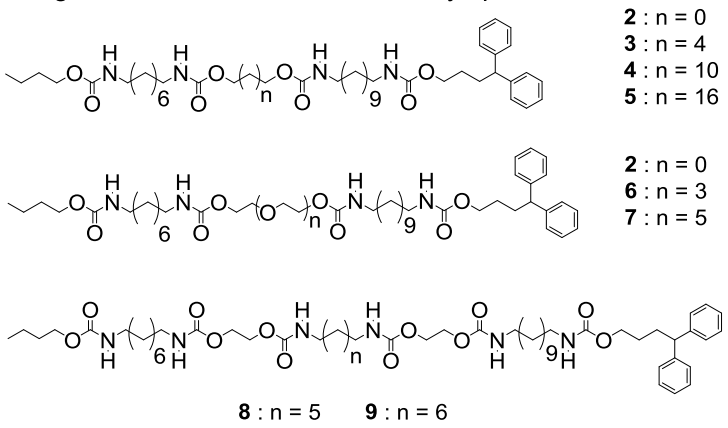

C. Oligocarbamate rods with bulky spacers
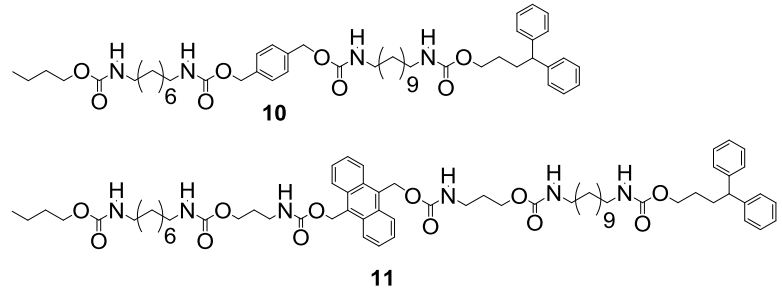

D. Single station rods
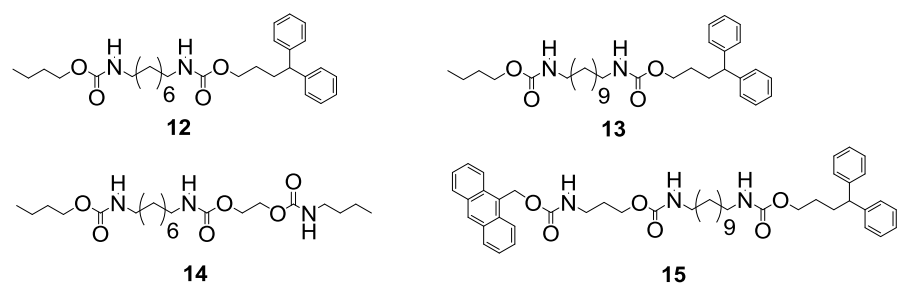

Figure 2. Formula of aromatic oligoamide host 1 and of the various rod-like guests used in this study.

Structural insights were also gathered in the solid state. Crystals of (1) $\supset$ $\supset \mathbf{1 2}$ and (1) $\supset \mathbf{1 3}$ were obtained from the slow diffusion of $n$-hexane in a chloroform solution of each complex.
A close-up look at the two crystal structures confirmed different extents of the relative screwing of the two strands in each double helix. ${ }^{9 b, c}$ This allows $(\mathbf{1})_{2}$ to accommodate guests of different 
lengths by adjusting the positions of the pyridine trimer pinchers so that they match with the positions of the carbonyl groups of the rod (Figures 3D,E). In solution, an indicator of the variable positioning of the two strands of $\mathbf{1}$ is the different chemical shift values of the protons in position 4 of the 2,6-pyridinedicarboxamide units which are found near $8.8 \mathrm{ppm}$ in $(\mathbf{1})_{2} \supset \mathbf{1 3}$ and below 8.4 ppm in (1) ( $_{2} \mathbf{1 2}$ (Figures S3 vs. S4, see also Figure $4 \mathrm{~A} v s .4 \mathrm{C}$ and Figure 2 in reference $9 \mathrm{~b}$ ). One may note that the terminal diamino pyridine unit of each strand is flipped out in the structure of $(\mathbf{1})_{2} \supset \mathbf{1 2}$. This has been observed previously in other duplexes involving terminal pyridine units even in the absence of guest. ${ }^{9 a, 12}$
A.

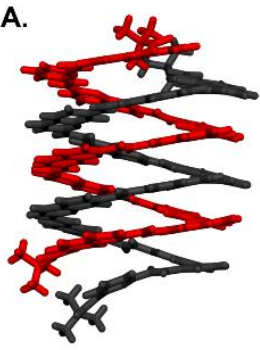

B.

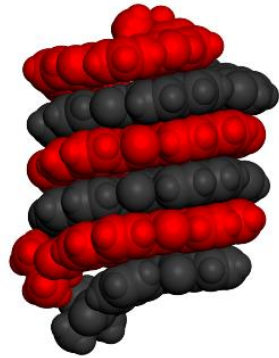

C.

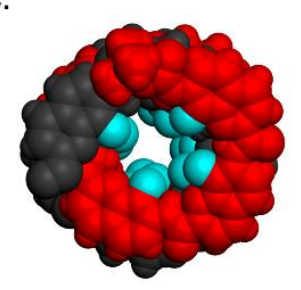

G.

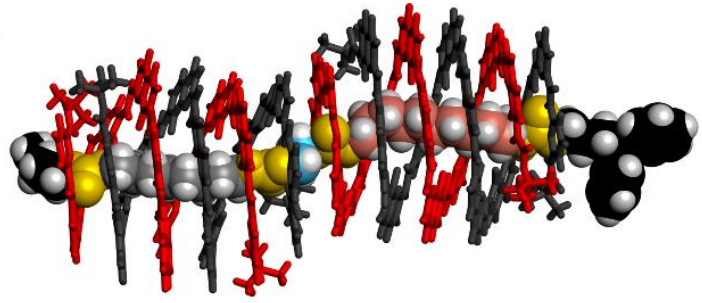

H.

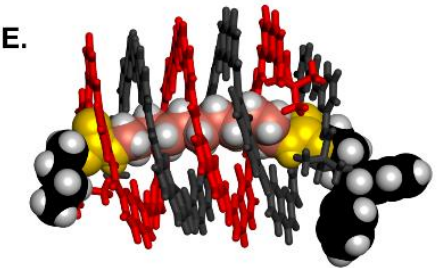

carbamate

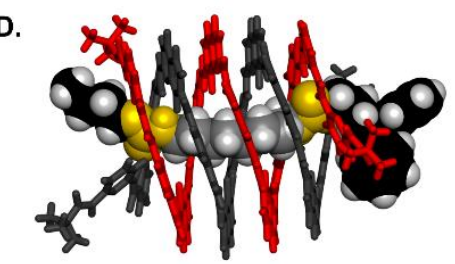

anchor points

F.

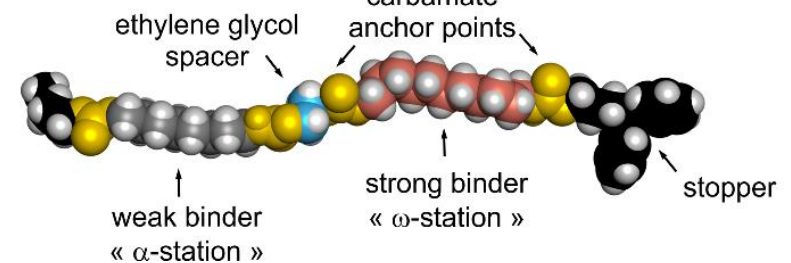

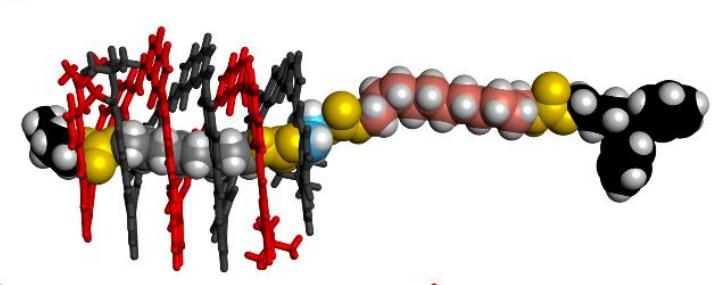

I.

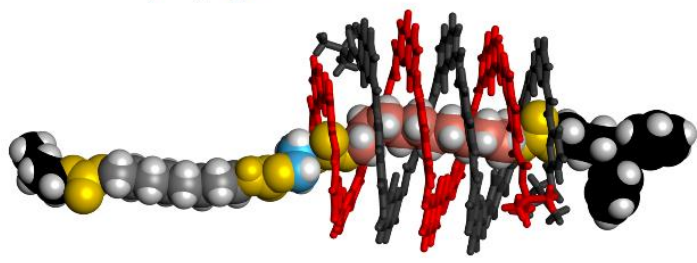

Figure 3. Structures in the solid state analyzed by single crystal $x$-ray crystallography of the antiparallel double helix of (1) 2 and of its complexes with various oligocarbamate rods. (A-C) structure of (1)2: side views in tube (A) and CPK (B) representations and top view (C) in CPK representation. In (C), terminal pyridine trimers have been deleted to highlight the cavity of the helix; fluorine atoms are shown in light blue color. (D) Structure of the [3]foldaxane (1) $\supset \mathbf{1 2}$ in tube (double helix) and CPK (rod) representations. (E) Structure of the [3]fold-

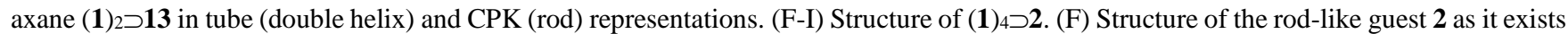
in [5]foldaxane (1) $)_{4} \supset \mathbf{2}$. (G) Side view of (1) $)_{4} \supset \mathbf{2}$. The guest and the helices appear in CPK and tube representations, respectively. In (H) and (I) one double helix of (1) $)_{4} \supset \mathbf{2}$ has been omitted on the strong binder or the weak binder stations, respectively to illustrate possible structures of (1) $2 \supset \mathbf{2} \alpha$ and (1) $)_{2} \supset \mathbf{2} \omega$, respectively. Only $P$ helical enantiomers are shown. The structures belong to non-Sohncke (enantiogenic) space groups and thus also contain $M$ enantiomers. Isobutoxy side chains and included solvent molecules have been omitted for clarity.

Directional sliding motion in double station rods. Foldaxane formation was then investigated with double station rods 2-7 (Figure 1A and 2B). These rods all bear both a weak binding station ( $\alpha$-station, 8 methylenes as in 12) and a strong binding station ( $\omega$-station, 11 methylenes as in $\mathbf{1 3}$ ). In the parent compound of the series, guest $\mathbf{2}$, the two stations are separated by a single ethylene glycol unit (Figure 3F). Upon adding an excess ${ }^{13}$ of rod 2 (10 equiv. with respect to $\left.(\mathbf{1})_{2}\right)$ to a $\mathrm{CDCl}_{3}$ solution of antiparallel $(\mathbf{1})_{2}$ NMR showed that a single new species was immediately and quantitatively formed that corresponds to the fast and quantitative threading of $(\mathbf{1})_{2}$ on $\mathbf{2}$ (Figure $4 \mathrm{~B}$ ). Intense nOe correlations between the bound rod and the double helix clearly indicate that $(\mathbf{1})_{2}$ is then located on the $\alpha$-station (Figure S26). This [3]foldaxane was named (1) $)_{2} \supset \mathbf{2} \alpha$ in reference to the weak binding $\alpha$-station and represents a kinetically favored intermediate: the helix does not immediately reach the more favorable $\omega$-station. Upon standing at $17^{\circ} \mathrm{C},(\mathbf{1})_{2} \supset \mathbf{2} \alpha$ disappeared over the course of days and was replaced by another foldaxane species (Figure 4C-E) reflecting that $(\mathbf{1})_{2} \supset \mathbf{2} \alpha$ is not the most stable complex. The new species was assigned to be $(\mathbf{1})_{2} \supset \mathbf{2} \omega$ in which the double helix $(\mathbf{1})_{2}$ is positioned on the strong binding station near the bulky stopper. Most amide ${ }^{1} \mathrm{H}$ NMR resonances are slightly downfield shifted in (1) $\supset 2 \mathbf{2} \omega$ with respect to (1) ${ }_{2} \supset \mathbf{2} \alpha$ which is consistent with chemical shift differences between the resonances of $(\mathbf{1})_{2} \supset \mathbf{1 2}$ and (1) $\supset \mathbf{1 3}$ (Figure S5). The equilibrium was reached after almost 3 days and the final ratio $\omega / \alpha$ was found to be $75 / 25$ (Table 1 ) as expected from the ratio of association constants with $\mathbf{1 2}$ and $\mathbf{1 3}$.

$\mathrm{X}$-ray-quality single crystals were obtained by slow diffusion of $n$-hexane into a $\mathrm{CDCl}_{3}$ solution of $(\mathbf{1})_{2}$ and $\mathbf{2}$ at thermodynamic equilibrium. Unexpectedly, despite the use of an excess of guest 2 to favor the crystallization of the [3]foldaxane $(\mathbf{1})_{2} \supset \mathbf{2} \omega$, the resolution of the solid-state structure revealed a five component assembly made of two double helices threaded on rod 2 (Figure 3G-I). This structure confirms that $(\mathbf{1})_{2}$ can complex both stations of $\mathbf{2}$ and provides an illustration of what both (1) $)_{2} \supset \mathbf{2} \omega$ (Figure 3I) and (1) $)_{2} \supset \mathbf{2} \alpha$ (Figure 3H) may look 
like. The crystallization of [5]foldaxane (1) $\supset \mathbf{2}$ may have two non-exclusive explanations: (i) the undetected selective crystallization or precipitation of excess $\mathbf{2}$, if it occurs first, will enrich the mixture in (1) $)_{4} \supset \mathbf{2}$; (ii) (1) $)_{4} \supset \mathbf{2}$ may be much less soluble and
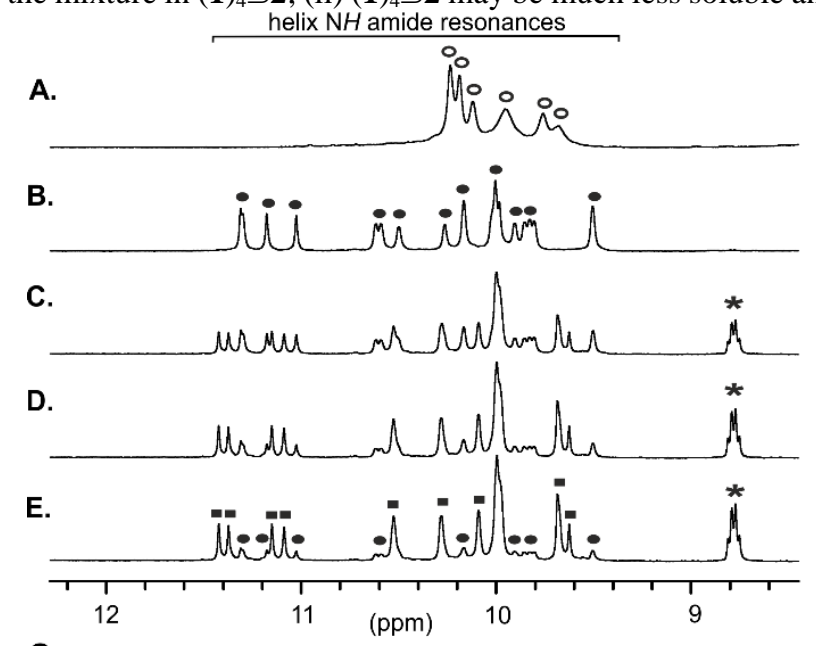

G.
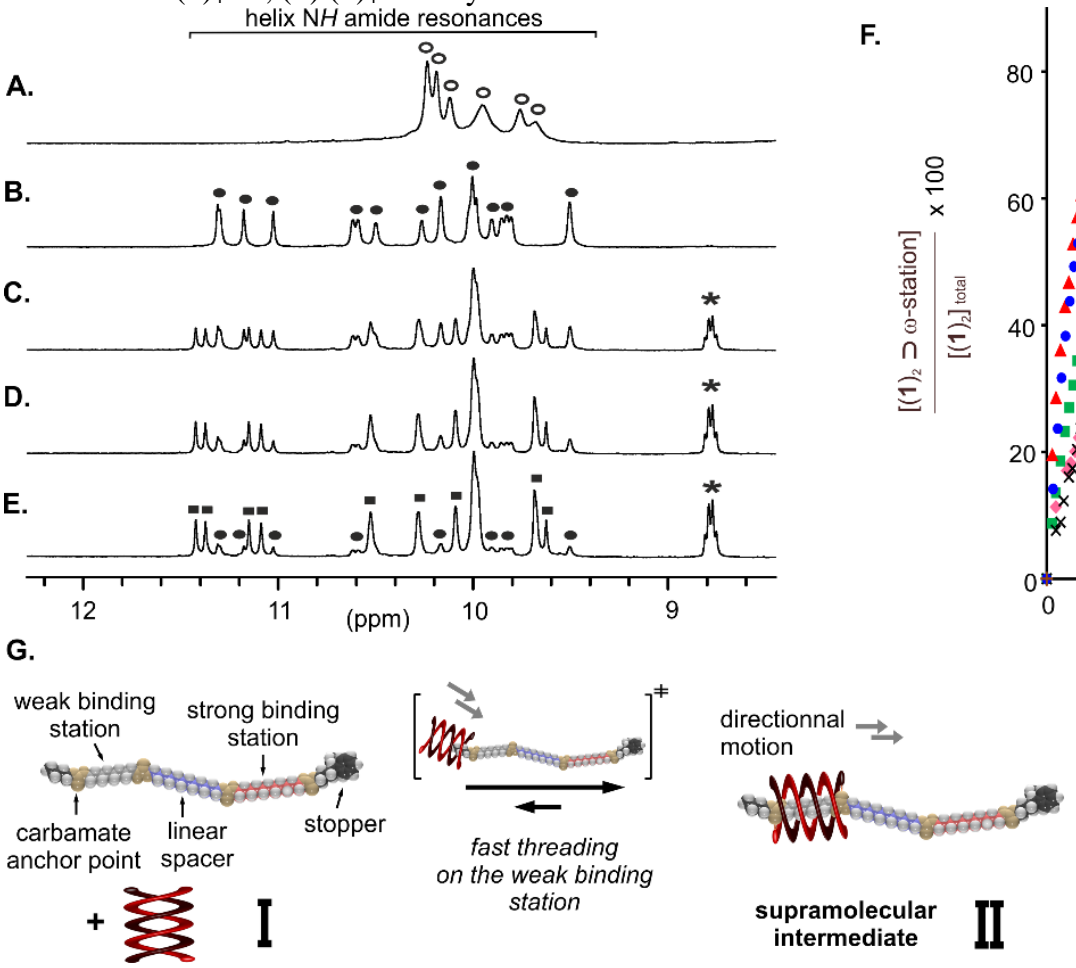

much more prone to crystallization than $(\mathbf{1})_{2} \supset \mathbf{2} \omega$, and may form crystals first even when it represents a minor component of the crystallization mixture.

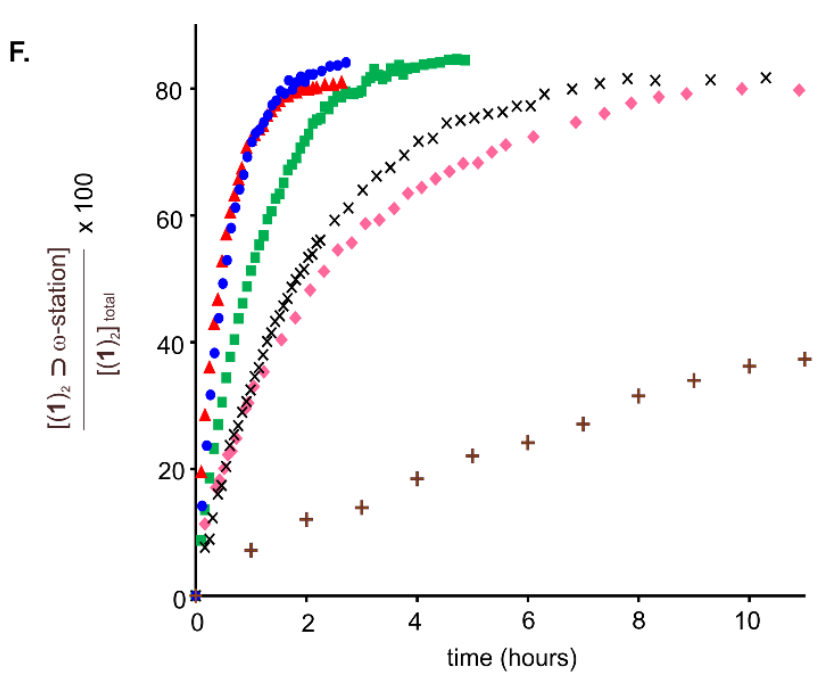

Figure 4. Kinetic and thermodynamic characterization of foldaxane formation between $(\mathbf{1})_{2}$ and double station rods 2-7. (A-E) Time dependent ${ }^{1} \mathrm{H}$ NMR spectra (400 MHz in $\mathrm{CDCl}_{3}$ at $290 \mathrm{~K}$, aromatic amide resonances region) showing the threading of $\mathbf{2}$ into $(\mathbf{1})_{2}$ to form (1) ${ }_{2} \supset \mathbf{2} \alpha$ and then (1) $\supset 2$. (A) initial antiparallel (1) 2 (2 mM); (B) 15 min. after the addition of 2 (20 mM), then after (C) 20 hours, (D) 40 hours, (E) 56 hours at equilibrium. Resonances of the free double helix $(\mathbf{1})_{2}$ are denoted with empty circles. Black circles and black squares indicate signals of (1) $)_{2} \supset \mathbf{2} \alpha$ and (1) $)_{2} \supset \mathbf{2} \omega$, respectively. Stars denote aromatic resonances (protons in position 4 of 2,6-pyridine dicarboxamide rings). (F) Time traces of threading and sliding motion of $(\mathbf{1})_{2}$ on rods $\mathbf{2}(+), \mathbf{3}(\mathbf{\Delta}), \mathbf{4}(\times), \mathbf{5}(\diamond), \mathbf{6}(\bullet)$ and $\mathbf{7}(\mathbf{\bullet})$. The concentration of host on each station was obtained through the integration of ${ }^{1} \mathrm{H}$ NMR amide resonances. (G) Schematic representation of the unidirectional sliding of the double helix along a double station rod.

Table 1. Kinetic data for the sliding of the investigated complexes determined by ${ }^{1} \mathrm{H}$ NMR.

\begin{tabular}{|c|c|c|c|c|c|c|c|c|c|c|c|}
\hline [3]foldaxane & $(\mathbf{1})_{2} \supset 2$ & $(\mathbf{1})_{2} \supset \mathbf{3}$ & $(\mathbf{1})_{2} \supset 4$ & $(\mathbf{1})_{2} \supset \mathbf{5}$ & $(1)_{2} \supset 6$ & $(1)_{2} \supset 7$ & $(\mathbf{1})_{2} \supset \mathbf{8}$ & $(\mathbf{1})_{2} \supset \mathbf{9}$ & $(\mathbf{1})_{2} \supset \mathbf{1 0}$ & $(\mathbf{1})_{2} \supset 11$ & (1) \\
\hline$k\left(10^{-5} \mathrm{~s}^{-1}\right)^{\mathrm{a}}$ & 1.2 & 37.3 & 11.3 & 9.7 & 36.3 & 23.0 & n. d. & n. d. & n. d. & n. d. & n. d. \\
\hline$\tau^{\mathrm{b}}$ (hours) & 18.0 & 0.44 & 1.82 & 2.12 & 0.50 & 0.96 & 37.0 & 102 & 122.4 & 117.8 & $97.9^{c}$ \\
\hline $\begin{array}{l}\% \text { of }(\mathbf{1})_{2} \text { on the } \omega \text {-sta- } \\
\text { tion at equilibrium }\end{array}$ & 75 & 81 & 82 & 81 & 83 & 85 & 80 & 64 & 76 & 76 & $69^{d}$ \\
\hline $\begin{array}{l}\text { number of atoms in the } \\
\text { spacer }\end{array}$ & 2 & 6 & 12 & 18 & 11 & 17 & 18 & 19 & n. a. & n. a. & n. a. \\
\hline
\end{tabular}

${ }^{a}$ pseudo-first order kinetic constants $k$ were calculated based on the integration of the ${ }^{1} \mathrm{H}$ NMR amide resonances corresponding to each complex $(\mathbf{1})_{2} \supset \mathbf{2} \alpha$ and $(\mathbf{1})_{2} \supset \mathbf{2} \omega$. The slope extracted from the plot of $-\ln \left(\left[(\mathbf{1})_{2} \supset \mathbf{2} \alpha\right] /\left[(1)_{2}\right]_{\text {total }}\right)$ as function of time corresponds to the kinetic constant of the sliding process. Calculation was performed during the initial phase of the helix motion for which the back sliding can be considered negligible; ${ }^{\mathrm{b}}$ time required to reach $50 \%$ of $(\mathbf{1})_{2}$ on the $\omega$-station corresponding to $\left[(\mathbf{1})_{2} \supset \omega\right.$-station] $=1 \mathrm{mM} ;{ }^{\mathrm{c}}$ competition experiment between $(\mathbf{1})_{2} \supset \mathbf{1 2}$ and $(\mathbf{1})_{2} \supset \mathbf{1 5}$. Double helix $(\mathbf{1})_{2}$ is already threaded on $\mathbf{1 2}$ prior to the addition of a stoichiometric amount of $\mathbf{1 5} ;{ }^{d}$ proportion of $(\mathbf{1})_{2}$ on rod $\mathbf{1 5}$. $n$. d.: not determined; n. a.: not applicable. 
A.

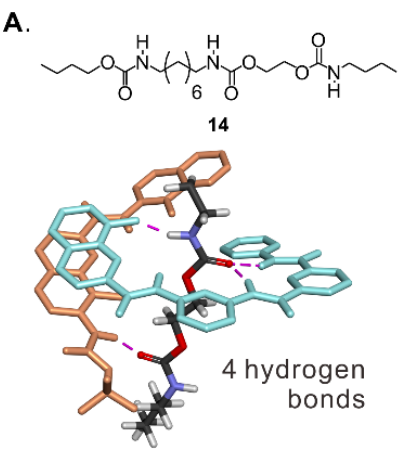

B.
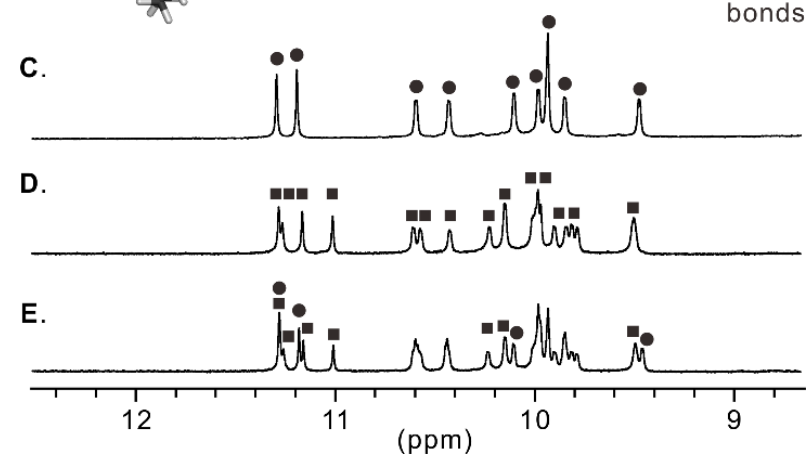

(ppm)

Figure 5. (A) Formula of rod 14 (top) and zoom on the binding pattern between rod $\mathbf{1 4}$ and the two strands of $(\mathbf{1})_{2}$ in an energy minimized model of (1) $\supset \mathbf{1 4}$ obtained by molecular mechanics (minimization with MMFFs force field) using Maestro v.6.5 (bottom); (B) Formula of rod $\mathbf{1 2}$ (top) and zoom on the binding pattern between rod $\mathbf{1 2}$ and the two strands of $(\mathbf{1})_{2}$ as observed in the crystal structure (1) ${ }_{2} \supset \mathbf{1 2}$ (bottom). The two strands of (1) 2 are shown in tube representation and are colored in either salmon or light blue. The rod is shown in tube representation and color coding is as follow: carbon (grey), nitrogen (blue), oxygen (red) and hydrogen (white). Hydrogen bonds are shown in magenta. Excerpt of the ${ }^{1} \mathrm{H}$ NMR spectra ( $400 \mathrm{MHz}, \mathrm{CDCl}_{3}, 298 \mathrm{~K}$ ) of the amide region of 1 (2 $\mathrm{mM}$ ) in the presence of: (C) 2 equiv. of rod 12; (D) 2 equiv. of rod 14 and (E) 2 equiv. of both 12 and 14.

Based on the experiments described above, the mechanism (sliding or winding/unwinding) of the transformation of $(\mathbf{1})_{2} \supset \mathbf{2} \alpha$ into $(\mathbf{1})_{2} \supset \mathbf{2} \omega$ cannot be ascertained. However, sliding above an ethylene glycol spacer has been established previously $^{9 a, b}$ and there is no reason to dismiss it in this case (see the subsequent section for experimental proof that sliding prevails here as well). NMR monitoring then provides an estimate of the sliding rate. Assuming that pseudo-first order kinetics apply when sliding starts, i.e. when the reverse sliding back to (1) $)_{2} \supset \mathbf{2} \alpha$ is negligible because there is little (1) $\supset \mathbf{2} \omega$ formed, a pseudo-first order kinetic constant was calculated to be $k=1.2$ $10^{-5} \mathrm{~s}^{-1}$ (Table 1, Figure 1 and Figure S6-S7).

We then examined the effect of elongating the central ethylene glycol spacer into longer alkanediols on the sliding rate of $(\mathbf{1})_{2}$. Sliding along $\mathbf{3}, \mathbf{4}$ and $\mathbf{5}$ proceeded as it did along $\mathbf{2}$, with the quick formation of a complex on the $\alpha$ station followed by the slow migration of $(\mathbf{1})_{2}$ to the $\omega$ station (Figure $\left.4 \mathrm{G}\right)$. Pseudofirst order rate constants were calculated (Table 1). In each of this three rods the spacer length was elongated to reach 6,12 and 18 methylenes, respectively. Yet, the sliding was found to be 30 times faster on rod 3 than on $\operatorname{rod} 2\left(k=37.310^{5} \mathrm{~s}^{-1}\right)$. Further elongation somewhat decelerated the motion but sliding remained 8 times faster on the longest rod $\mathbf{5}$ than on $\mathbf{2}$. This non monotonous trend was also observed upon elongating the central ethylene glycol into oligo-ethylene glycols: when compared to the short ethylene glycol spacer of $\mathbf{2}$, sliding rates along tetraethylene glycol (6) and hexaethylene glycol (7) were found to be higher by 30 and 20 fold, respectively. If one excludes 2 and its very short spacer, elongating spacers eventually slows down sliding but the attenuation of the rate constant is not pronounced. Furthermore, sliding along oligoethyleneglycols is faster than along alkanes, despite the preference of the former to adopt multiple gauche conformations that are a priori not compatible with the narrow cavity of $(\mathbf{1})_{2}$. For instance, sliding along rods 6 (resp. 7) was found to be 2-3 times faster than along 4 (resp. 5) despite their similar lengths (Table 1).

The distinctly slower sliding along $\mathbf{2}$ must in some manner relate to the proximity between the central carbamate functions of this rod. To explore what may slow down sliding from the $\alpha$ to the $\omega$-station of $\mathbf{2}$, we prepared rod $\mathbf{1 4}$ which possesses an $\alpha$ station plus a single carbamate group connected by an ethylene glycol (Figure 5A). The relative affinity of $\mathbf{1 4}$ for $(\mathbf{1})_{2}$ was assessed in a competition experiment: 12 and 14 were mixed in excess (2 equiv. each) with $(\mathbf{1})_{2}$. The ratio $\left[(\mathbf{1})_{2} \supset \mathbf{1 4}\right] /\left[(\mathbf{1})_{2} \supset \mathbf{1 2}\right]$ was calculated by integrating NMR signals and showed that the additional carbamate group of $\mathbf{1 4}$ is responsible for an enhancement of binding to $(\mathbf{1})_{2}$ by a factor of 1.7 with respect to $\mathbf{1 2}$ (Figure 5C-D). A comparison between the x-ray structure of $(\mathbf{1})_{2} \supset \mathbf{1 2}$ and an energy minimized molecular model of $(\mathbf{1})_{2} \supset \mathbf{1 4}$ revealed the presence of an additional hydrogen bond between the $\mathrm{NH}$ of the terminal 8-fluoroquinoline of $(\mathbf{1})_{2}$ and the extra carbamate carbonyl group at the extremity of 14 (Figure 5A,B). This interaction is possibly responsible for the higher binding to $\mathbf{1 4}$ and is unlikely to occur with spacers longer than an ethylene glycol between carbamate functions. Thus, the singular slow kinetics of sliding of $(\mathbf{1})_{2}$ along two station rod $\mathbf{2}$ might arise from a higher affinity $(\mathbf{1})_{2}$ with both the $\alpha$ - and $\omega$-stations as compared to rods in which the $\alpha$ - and $\omega$-stations are more distant.

Directional sliding motion in triple station rods. Rod design was taken one step further by placing an additional, $\beta$-binding station, in-between the $\alpha$ - and $\omega$-stations. Triple station rods $\mathbf{8}$ and 9 both possess the same $\alpha$ - and $\omega$-stations as double station rods. In rod $\mathbf{8}$, the central $\beta$-station has a weak affinity for antiparallel $(\mathbf{1})_{2}\left(K_{a}=1100 \mathrm{~L} \mathrm{~mol}^{-1}\right)$. In $\operatorname{rod} \mathbf{9}$, the central $\beta$-station is identical to the $\alpha$-station. All stations are separated by an ethylene glycol spacer. Threading experiments with rods $\mathbf{8}$ and 9 were analyzed by ${ }^{1} \mathrm{H}$ and ${ }^{19} \mathrm{~F}$ NMR. The determination of a pseudo-first order rate constant was not as straightforward as for the other rods due to binding to, and reverse sliding from, the $\beta$-station. Instead, to compare with the others rods we monitored for each foldaxane the time required to reach $50 \%$ occupancy of $(\mathbf{1})_{2}$ on the $\omega$-station ( $\tau$ in Table 1$)$. When compared to rods 3-7, sliding kinetic of $(\mathbf{1})_{2}$ to reach equilibrium (Table 1) was found to be at least 17 times slower. Both rods 8 and 9 showed a much longer sliding time ( $\tau=37$ and 102 hours, respectively) to join the terminal $\omega$-station. As expected, the final percentage of double helix on the terminal $\omega$-station of 9 was found to be lowered by the competition binding between the $\omega$ and both $\alpha$ - and $\beta$-stations. In the case of $\operatorname{rod} \mathbf{8}$, at thermodynamic equilibrium, the final ratio was found to be only slightly altered due to weak competition of the intermediate binding site with the $\omega$-station. 
In summary of the two sections above, when $(\mathbf{1})_{2}$ is mixed with rods having two or three binding stations separated by linear non-hindered alkyl or ethylene glycol spacers, the quick formation of a kinetically favored intermediate is observed, followed by a slower migration to the best binding station. The kinetics of the overall process much depends on what is between the $\alpha$ - and $\omega$-stations (length and chemical nature of the spacer, additional station), which is consistent with the hypothesis that the helix actually slides along the molecular track from the unhindered end to the bulkier side of the rod.

Threading-sliding vs. winding-unwinding mechanisms. To ascertain the sliding mechanism hypothesized above and to promote an alternate supramolecular pathway, we introduced double station rods $\mathbf{1 0}$ and $\mathbf{1 1}$ in which the spacers separating the two stations contain a bulky component (Figure 2C), either benzene or anthracene, that are a priori too large to pass through the cavity of $(\mathbf{1})_{2}$ without a major distortion. In these designs, the helix may quickly thread onto the $\alpha$-station, but reaching the thermodynamically favored $(\mathbf{1})_{2} \supset \mathbf{1 0} \omega$ and $(\mathbf{1})_{2} \supset \mathbf{1 1} \omega$ complexes requires a winding of the helix around the $\omega$-station, presumably after its dissociation from the $\alpha$-station. Self-assembly processes were monitored as for other rods. As expected, upon mixing 10 with $(\mathbf{1})_{2}$, a kinetically favored species assigned to (1) $)_{2} \supset \mathbf{1 0} \alpha$ is quickly observed (Figure 6A,B). The emergence of a new set of resonances corresponding to $(\mathbf{1})_{2} \supset \mathbf{1 0} \omega$ was considerably slower than with non-bulky spacers. Again, to compare the motion kinetics of $(\mathbf{1})_{2}$ on rod equipped with bulky spacers

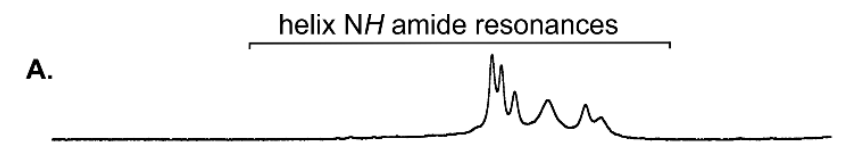

B.

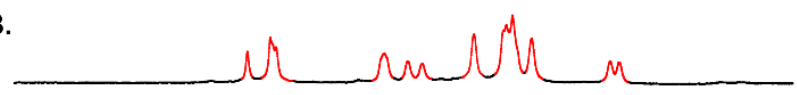

C.

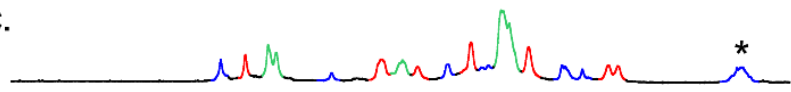

D.

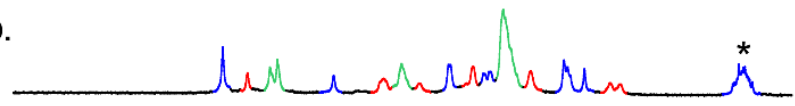

E.

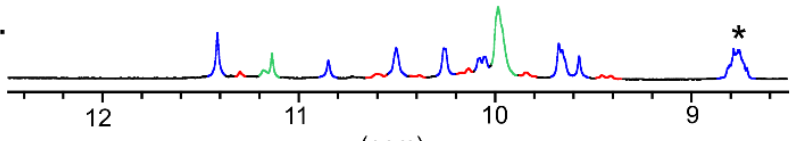

G.

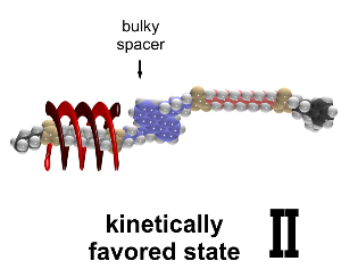

(ppm)
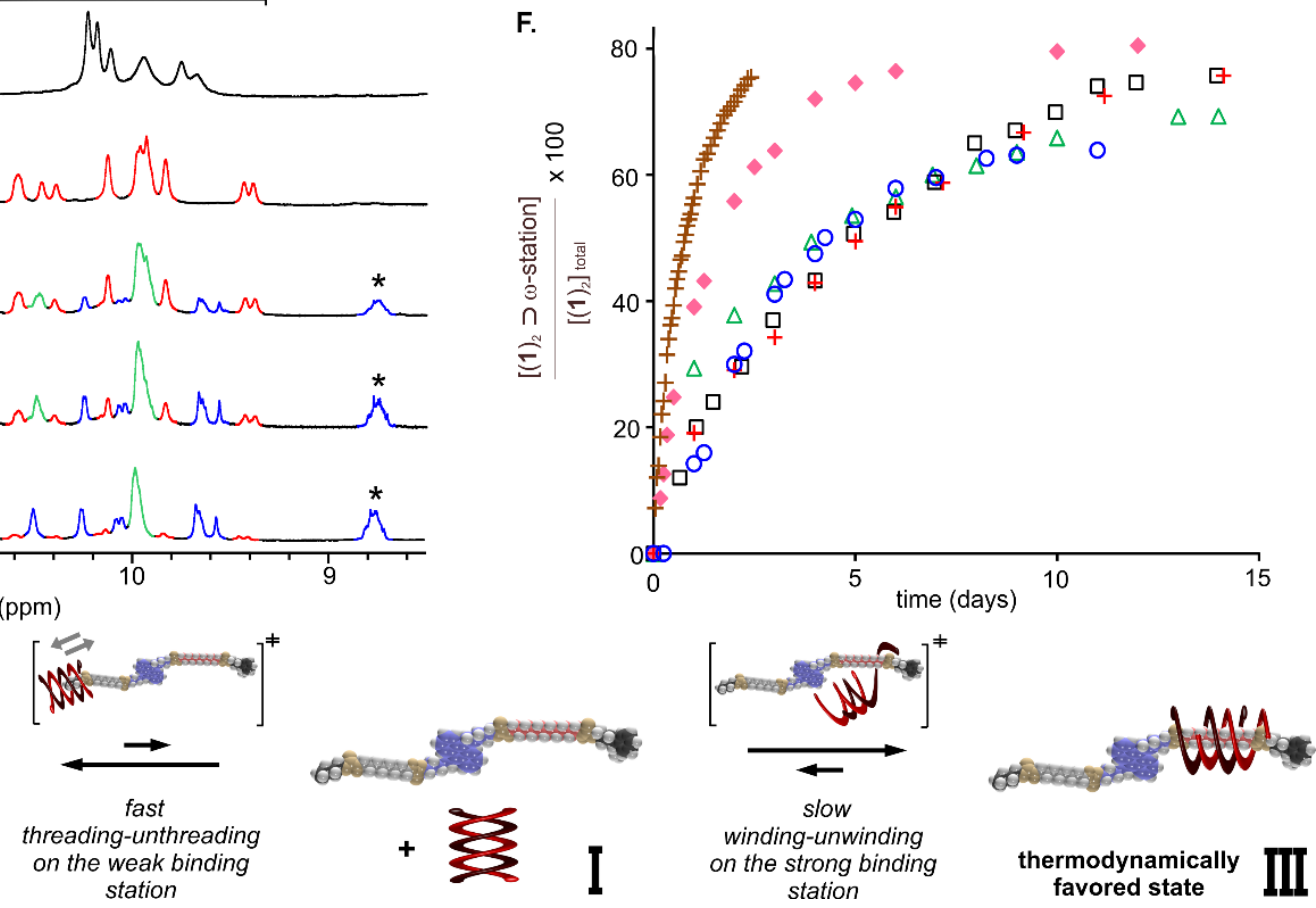

Figure 6. Kinetic and thermodynamic characterization of foldaxane formation between $(\mathbf{1})_{2}$ and triple and double station rods 8-11. (A-E) Time dependent ${ }^{1} \mathrm{H}$ NMR spectra $\left(400 \mathrm{MHz}_{\text {in }} \mathrm{CDCl}_{3}\right.$ at $290 \mathrm{~K}$, aromatic amide resonances region) showing the fast threading of $\mathbf{1 0}$ into $(\mathbf{1})_{2}$ to form (1) $\supset \mathbf{1 0} \alpha$ and the slow conversion into (1) $\supset \mathbf{1 0} \omega$. (A) ${ }^{1} \mathrm{H}$ NMR spectra of free $(\mathbf{1})_{2}(2 \mathrm{mM})$; (B) 10 min. after the addition of $\mathbf{1 0}$ (20 $\mathrm{mM}$ ), then after (C) 2 days, (D) 5 days and (E) 15 days at equilibrium. Black colored signals are those of the free (1) 2 whereas red and blue resonances belong to $(\mathbf{1})_{2} \supset \mathbf{1 0} \alpha$ and $(\mathbf{1})_{2} \supset \mathbf{1 0} \omega$, respectively. Green resonances were not assigned due to signal overlap. Stars denote aromatic resonances. (F) Time traces of the conversion of $\alpha$ into $\omega$ complexes of $(\mathbf{1})_{2}$ on rods $2(+), \mathbf{8}(\diamond), \mathbf{9}(0), \mathbf{1 0}(+)$ and $\mathbf{1 1}(\square)$; unfolding-refolding of $(\mathbf{1})_{2}$ from rod 12 to $15(\triangle)$. Concentration of host on each station was assessed by ${ }^{1} \mathrm{H}$ NMR through the integration of the amide resonances 
of the double helix. (G) Schematic representation of the overcoming of a bulky obstacle by a double helix through an unwinding-rewinding mechanism.

\section{CONCLUSION}

Our results demonstrate how a clear sequence of events can be designed in a complex supramolecular system by exploiting the different time scales on which each of these events takes place. The anti-parallel form of dimer $(\mathbf{1})_{2}$ was isolated by precipitation and then trapped in solution in foldaxane structures upon binding to rod-like guests before the parallel form of $(\mathbf{1})_{2}$ had time to appear. Rod-like guest binding can be made to occur selectively through the kinetically favored less hindered end of the rod to produce a foldaxane on the first binding station, even though thermodynamically preferred binding stations may be available in the system. Once threading of the rod into $(\mathbf{1})_{2}$ is achieved, sliding of $(\mathbf{1})_{2}$ along the rod takes place at rates substantially faster than helix dissociation and re-association through an unwinding-rewinding mechanism. The sliding direction along the rod can be biased by installing a gradient of binding stations of increasing thermodynamic stability. Along the sliding path, small barriers can be installed such as long segments deprived of binding stations that do not significantly hamper motion. However, if a sufficiently large barrier is placed on the rod, sliding is blocked at the binding station that precedes the barrier and a slower dissociation and re-association through an unwinding-rewinding mechanism is imposed for $(\mathbf{1})_{2}$ to reach a thermodynamically more favorable station placed further on the rod.

These various kinetically controlled pathways illustrate the possible error correction mechanisms in the formation of multifoldaxanes from the loading of several foldamer helices onto multistation rods. ${ }^{9 \mathrm{c}}$ Further elaboration of controlled oriented foldaxane motions include the design of helical systems having different ends that can form a foldaxane only by threading the rod through one end and not the other. Such foldaxanes would thus have a front that can move towards one extremity of a rod and a back that can move towards the other extremity of the rod. Progress along these is currently being made in our laboratories and will be reported in due course.

\section{ASSOCIATED CONTENT}

\section{Supporting Information}

The Supporting Information is available free of charge on the ACS Publications website at DOI:

Experimental details for synthetic procedures, spectroscopic data (PDF).

Crystallographic information files for $(\mathbf{1})_{2},(\mathbf{1})_{2} \supset \mathbf{1 2},(\mathbf{1})_{2} \supset \mathbf{1 3}$, and (1) $4 \supset 2$ (CIF).

\section{AUTHOR INFORMATION}

\section{Corresponding Authors}

*y.ferrand@iecb.u-bordeaux.fr

*i.huc@iecb.u-bordeaux.fr

\section{Present Address}

$\uparrow$ Department of Chemical Technology of Drugs, Poznan University of Medical Sciences, Grunwaldzka 6, 60-780 Poznan, Poland.

\section{Notes}

The authors declare no competing financial interest.

\section{ACKNOWLEDGMENT}

This work was supported by the China Scholarship Council (predoctoral fellowship to X.W.) and by the European Research Council under the European Union's Seventh Framework Programme (grant agreement no. ERC-2012-AdG-320892, post-doctoral fellowship to B.W.). The authors thank Brice Kauffmann (IECBUMS 3033) for his help during data collection and resolution of the crystal structures.

\section{REFERENCES}

(1) (a) Mathias, J. P.; Seto, C. T.; Simanek, E. E.; Whitesides, G. M. J. Am. Chem. Soc. 1994, 116, 1725-1735. (b) Prins, L. J.; De Jong, F.; Timmerman, P.; Reinhoudt, D. N. Nature 2000, 408, 181-184. (c) Tashiro, S.; Tominaga, M.; Kusukawa, T.; Kawano, M.; Sakamoto, S.; Yamaguchi, K.; Fujita M. Angew. Chem. Int. Ed. 2003, 42, 3267-3270. (d) Badjic, J. D.; Cantrill, S. J.; Stoddart, J. F. J. Am. Chem. Soc. 2004, 126, 2288-2289. (e) Lohr, A.; Lysetska, M.; Würthner, F. Angew. Chem. Int. Ed. 2005, 44, 5071-5074. (f) Oshikiri, T.; Takashima, Y.; Yamaguchi, H.; Harada A. J. Am. Chem. Soc. 2005, 127, 12186-12187. (g) Mukhopadhyay, P.; Zavalij, P. Y.; Isaacs L. J. Am. Chem. Soc. 2006, 128, 14093-14102. (h) de Greef, T. F. A.; Ligthart, G. B. W. L.; Lutz, M.; Spek, A. L.; Meijer, E. W.; Sijbesma, R. P. J. Am. Chem. Soc. 2008, 130, 5479-5486. (i) Cangelosi, V. M.; Carter, T. G.; Zakharov, L. N.; Johnson, D. W. Chem. Commun. 2009, 5606-5608. (j) Chapin, J. C.; Kvasnica, M.; Purse, B. W. J. Am. Chem. Soc. 2012, 134 15000-15009. (k) Gan, Q.; Ferrand, Y.; Chandramouli, N.; Kauffmann, B.; Aube, C.; Dubreuil, D.; Huc, I. J. Am. Chem. Soc. 2012, 134, 15656-15659. (1) Danylyuk, O.; Fedin, V. P.; Sashuk, V. Chem. Commun., 2013, 49, 1859-1861.

(2) Erbas-Cakmak, S.; Leigh, D. A.; McTernan, C. T.; Nussbaumer, A. L. Chem. Rev. 2015, 115, 10081-10206.

(3) Vale, R. D.; Reese, T. S.; Sheetz, M. P. Cell 1985, 42, 39-50.

(4) For recent reviews on artificial molecular walkers see: (a) von Delius, M.; Leigh D. A. Chem Soc. Rev. 2011, 40, 3656-3676. (b) Pan, J.; Li, F.; Cha, T. G.; Chen, H.; Choi, J. H. Curr. Opin. Biotechnol. 2015, 34, 56-64.

(5) (a) Sherman, W. B.; Seeman N. C. Nano Letters 2004, 4, 1203-1207. (b) Kwon, K.-Y.; Wong, K. L.; Pawin, G.; Bartels, L.; Stolbov, S.; Rahman T. S. Phys. Rev. Lett. 2005, 95, 166101-116104. (c) von Delius, M.; Geertsema, E. M.; Leigh, D. A.; Tang, D. T. D. J. Am. Chem. Soc. 2010, 132, 16134-16145. (d) von Delius, M.; Geertsema, E. M.; Leigh, D. A. Nat. Chem. 2010, 2, 96-101. (e) Barrell, M. J.; Campana, A. G.; von Delius, M.; Geertsema, E. M.; Leigh D. A. Angew. Chem. Int. Ed. 2011, 50, 285-290. (f) Perl, A.; Gomez-Casado, A.; Thompson, D.; Dam, H. H.; Jonkheijm, P.; Reinhoudt, D. N.; Huskens, J. Nat. Chem. 2011, 3, 317-322. (g) Kovaricek, P.; Lehn, J.-M. J. Am. Chem. Soc. 2012, 134, 9446-9455. (h) Campana, A. G.; Carlone, A.; Chen, K.; Dryden, D. T. F.; Leigh, D. A.; Lewandowska, U.; Mullen K. M. Angew. Chem. Int. Ed. 2012, 51, 5480-5483. (i) Campana, A. G.; Leigh, D. A.; Lewandowska U. J. Am. Chem. Soc. 2013, 135, 8639-8645. (j) Beves, J. E.; Blanco, V.; Blight, B. A.; Carrillo, R.; D'Souza, D. M.; Howgego, D.; Leigh, D. A.; Slawin, A. M. Z.; Symes M. D. J. Am. Chem. Soc. 2014, 136, 2094-2100. (k) Pulcu, G. S.; Mikhailova, E.; Choi, L. S.; Bayley H. Nat. Nanotechnol. 2014, 10, 76-83. (1) Kovaricek, P.; Lehn, J.-M. Chem. Eur. J. 2015, 21, 9380-9384.

(6) (a) Harada, A.; Li, J.; Kamachi M. Nature 1992, 356, 325-327. (b) Herrmann. W.; Keller, B.; Wenz G. Macromolecules 1997, 30, 4966-4972.

(7) (a) Coumans, R. G. E.; Elemans, J. A. A. W.; Nolte, R. J. M.; Rowan. A. E. Proc. Natl. Acad. Sci. U.S.A. 2006, 103, 19647-19651; (b) Deutman, A. B. C.; Monnereau, C. ; Elemans, J. A. A. W.; Ercolani, G.; Nolte, R. J. M.; Rowan, A. E. Science 2008, 322, 1668-1671; (c) Deutman, A. B. C.; Cantekin, S.; Elemans, J. A. A. W.; Rowan, A. E.; Nolte, R. J. M. J. Am. Chem. Soc. 2014, 136, 9165-9172. (d) Cantekin, S.; Markvoort, A. J.; Elemans, J. A. A. W.; Rowan, A. E.; Nolte R. J. M. J. Am. Chem. Soc. 2015, 137, 3915-3923. (e) Peck, E. 
M.; Liu, W.; Spence, G. T.; Shaw, S. K.; Davis, A. P.; Destecroix, H.; Smith; B. D. J. Am. Chem. Soc. 2015 137, 8668-8671.(f) Liu, W.; Peck, E. M.; Hendzel, K. D.; Smith B. D. Org. Lett. 2015, 17, 5268 5271.

(8) (a) Thordarson, P.; Bijsterveld, E. J. A.; Rowan, A. E.; Nolte, R. J. M. Nature, 2003, 424, 915-918. (b) Takashima, Y.; Osaki, M.; Ishimaru, Y.; Yamaguchi, H.; Harada, A. Angew. Chem. Int. Ed. 2011, 50, 7524-7528. (c) Lewandowski, B.; Bo, G. D.; Ward, J. W.; Papmeyer, M.; Kuschel, S.; M. J. Aldegunde, Gramlich, P. M. E.; Heckmann, D.; Goldup, S. M.; D’Souza, D. M.; Fernandes, A. E.; Leigh, D. A. Science, 2013, 339, 189-193. (d) Bo, G. D.; Kuschel, S.; Leigh, D. A.; Lewandowski, B.; Papmeyer, M.; Ward, J. W. J. Am. Chem. Soc. 2014, 136, 5811-5814. (e) van Dongen, S. F. M.; Clerx, J.; Nørgaard, K.; Bloemberg, T. G.; Cornelissen, J. J. L.; Trakselis, M. A.; Nelson, S. W.; Benkovic, S. J.; Rowan, A. E.; Nolte R. J. M. Nat. Chem., 2013, 5, 945-951.

(9) (a) Gan, Q.; Ferrand, Y.; Bao, C.; Kauffmann, B.; Grélard, A.; Jiang, H.; Huc, I. Science 2011, 331, 1172-1175. (b) Ferrand, Y.; Gan, Q.; Kauffmann, B.; Jiang, H.; Huc, I. Angew. Chem. Int. Ed. 2011, 50, 7572-7575. (c) Gan, Q.; Wang, X.; Kauffmann, B.; Rosu, F.; Ferrand, Y.; Huc I. Nat. Nanotech. 2017, 12, 447-452. (d) Denisov, S. A.; Gan, Q.; Wang, X.; Scarpantonio, L.; Ferrand, Y.; Kauffmann, B.; Jonusauskas, G.; Huc, I.; McClenaghan, N. D. Angew. Chem. Int. Ed. 2016, 55, 1328-1333.
(10) (a) Nishinaga, T.; Tanatani, A.; Oh, K.; Moore, J. S. J. Am. Chem. Soc. 2002, 124, 5934-5935. (b) Tanatani, A., Hughes, T. S., Moore, J. S. Angew. Chem. Int. Ed. 2002, 41, 325-328. (c) Petitjean, A. Cuccia, L. A.; Schmutz, M.; Lehn, J. M. J. Org. Chem. 2008, 73, 2481-2495.

(11) (a) Xue, M.; Yang, Y.; Chi, X.; Yan, X.; Huang, F. Chem. Rev., 2015 , 115, 7398-7501. (b) Jiménez, M. C.; Dietrich-Buchecker, C; Sauvage, J.-P. Angew. Chem. Int. Ed. 2000, 39, 3284-3287. (c) Wu, J.; Leung, K. C. F.; Benitez, D.; Han, J. Y.; Cantrill, S. J.; Fang, L.; Stoddart J. F. Angew. Chem. Int. Ed. 2008, 47, 7470-7474. (d) Du, G.; Moulin, E.; Jouault, N., Buhler, E., Giuseppone, N. Angew. Chem. 2012, 124, 12672-12676. (e) Ragazzon, G.; Baroncini, M. Silvi, S.; Venturi, M.; Credi, A. Nat. Nanotech. 2014, 10, 70-75. (f) Wilson, M. R.; Solá, J.; Carlone, A.; Goldup, S. M.; Lebrasseur N.; Leigh, D. A. Nature 2016, 534, 235-240. (g) Waelès, P.; Riss-Yaw, B.; Coutrot, F. Chem. Eur. J. 2016, 22, 6837-6845. (g) Lin, Q.; Hou, X; Ke, C. Angew. Chem. Int. Ed. 2017, 56, 4452-4457.

(12) Berni, E.; Kauffmann, B.; Bao, C.; Lefeuvre, J.; Bassani, D. M.; Huc I. Chem. Eur. J. 2007, 13, 8463-8469.

(13) With excess rod, no measurable amount of the higher aggregate, [5]foldaxane, in which two double helices are bound to the rod, was observed.

\section{TOC GRAPHIC}

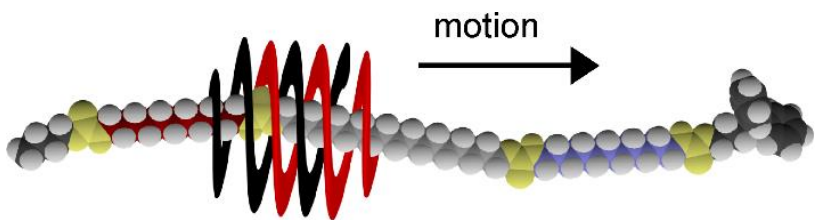

